\section{Riesgo de malaria en mujeres embarazadas de zonas de baja endemia de la Amazonia Peruana}

Cada año, más de 24 millones de mujeres resultan embarazadas en zonas endémicas de malaria y se encuentran en un mayor riesgo de infección, con graves consecuencias por las complicaciones que pueden sufrir tanto la madre como el feto. La infección por malaria durante el embarazo puede provocar el nacimiento de niños con bajo peso, el retraso en el crecimiento intrauterino del feto y la anemia materna, entre otros trastornos. Las mujeres embarazadas con relativamente baja inmunidad adquirida contra la malaria tienen un mayor riesgo de sufrir las complicaciones más graves, entre ellas la anemia grave por malaria, abortos, el nacimiento de un niño muerto $\mathrm{y}$, finalmente, la muerte de la madre y del recién nacido. El grado de endemicidad de la transmisión de la malaria, el nivel de inmunidad adquirida y el número de embarazos anteriores son algunos de los principales factores que influyen en la epidemiología de la malaria durante el embarazo.

La mayoría de los estudios realizados sobre la epidemiología y el efecto de la malaria durante el embarazo se han llevado a cabo en zonas de alta endemia, donde Plasmodium falciparum y P. vivax han sido los principales agentes infecciosos. Esos estudios han demostrado que las mujeres embarazadas tienen una mayor susceptibilidad a la malaria y a sus complicaciones que el resto de los adultos.

En este trabajo se estudió la epidemiología de la malaria por $P$. falciparum y $P$. vivax durante el embarazo en una zona de baja endemia en la región amazónica de Iquitos, Perú, durante 2004-2005.

En contraste con la mayoría de los estudios realizados en zonas de alta prevalencia, la información de la vigilancia pasiva en ocho postas sanitarias atendidas por el Ministerio de Salud de ese país demostró que las mujeres embarazadas presentaron una menor prevalencia de malaria que el resto de la población ( $7,5 \%$ en 2004 y 6,6\% en 2005 frente a $20,6 \%$ y $22,4 \%$ en la población total, respectivamente).

También se comparó la prevalencia en mujeres embarazadas con la encontrada en hombres y en mujeres no embarazadas de 14 a 45 años de edad que participaron en el estudio de seguimiento activo basado en la población en esa misma zona. En este caso, se encontró que la mujeres embarazadas tenían 2,3 veces mayor probabilidad de estar infec- tadas con el parásito $P$. falciparum que las mujeres no embarazadas del mismo grupo de edad (intervalo de confianza de $95 \%$ : 1,32 a $3,95, P=0,04)$. A pesar de que las mujeres embarazadas no demostraron un mayor riesgo de infección por $P$. vivax en comparación con las no embarazadas, la incidencia encontrada de cerca de $20 \%$ fue significativamente mayor que la encontrada como resultado de la vigilancia pasiva realizada por las postas sanitarias $(6,0-7,5 \%)$.

El análisis de los factores de riesgo demostró que las mujeres embarazadas con malaria eran de mayor edad y habían tenido más embarazos que las mujeres que no estaban embarazadas. Esto marca una diferencia radical con respecto a los estudios realizados en zonas endémicas, en los que se encontró que la resistencia a la malaria en estas mujeres aumentaba con la edad y el número de embarazos previos.

La mayor frecuencia de infección, tanto con $P$. vivax como con $P$. falciparum, encontrada durante el seguimiento activo en comparación con la encontrada en las postas sanitarias indica que las infecciones asintomáticas pueden ser frecuentes en las mujeres embarazadas. La infección por P. falciparum, incluso si es asintomática, tiene grandes implicaciones para la salud de las mujeres embarazadas y de su feto, ya que causa complicaciones más graves que la infección por $P$. vivax, como malaria cerebral, anemia grave por malaria e hipoglucemia, entre otras. Además, los eritrocitos infectados por $P$. falciparum pueden acumularse en la placenta y afectar a la transferencia de oxígeno entre la madre y el feto. Las infecciones asintomáticas pueden tener consecuencias mucho más graves en las mujeres embarazadas que en las no embarazadas, ya que pueden pasar inadvertidas y continuar causando los graves daños mencionados a la madre y al feto.

Este estudio demostró que en la vigilancia pasiva se subestimó la carga de la malaria en esta región y que la infección subclínica puede ser frecuente en las mujeres embarazadas. Además, las mujeres embarazadas en esta zona de bajo nivel de transmisión, donde el $P$. vivax es el agente causal dominante, corren un mayor riesgo por la infección por $P$. falciparum que por $P$. vivax.

La infección por malaria durante el embarazo, aun si es asintomática, es un grave problema de salud pública en Iquitos, Perú, y sus alrededores. Se deben realizar pruebas de diagnóstico para la mala- 
ria a todas las mujeres embarazadas que llegan a las postas de salud, incluso si antes reciben el diagnóstico de otra enfermedad asociada con el embarazo. Estos resultados justifican la realización de una investigación más detallada de las mujeres en el momento del parto para determinar los posibles desenlaces asociados con la infección por malaria y actuar en consecuencia. (Parekh FK, Hernández JN, Krogstad DJ, Martin Casapia W, Branch OH. Prevalence and risk of $P$. falciparum and $P$. vivax malaria among pregnant women living in the hypoendemic communities of the Peruvian Amazon. Am J Trop Med Hyg. 2007;77(3):451-7.) 